\title{
COHOMOLOGY OF A BOUNDING MANIFOLD
}

\author{
LARRY SMITH AND R. E. STONG
}

\begin{abstract}
This paper characterizes those subsets of $H^{*}(M ; Q)$ which are the image of the cohomology of some manifold whose boundary is $M$.
\end{abstract}

1. Introduction. The object of this note is to prove:

Proposition. Let $M^{n}$ be a closed oriented (or stably almost complex) manifold and $R^{*} \subset H^{*}(M ; Q)$. There is a compact oriented (or s.a.cx.) manifold with boundary $V$ so that $\partial V=M$ with $R^{*}=i^{*} H^{*}(V ; Q)$ where $i: M \rightarrow V$ is the inclusion if and only if $R^{*}$ satisfies:

(a) $R^{*}$ is a homogeneous subalgebra of $H^{*}(M ; Q)$ (i.e. $R^{*}$ is the direct sum of the groups $R^{i}=R^{*} \cap H^{i}(M ; Q)$ );

(b) $R^{*}$ contains the characteristic ring $\tau_{M}^{*} H^{*}(B S O ; Q)\left(\right.$ or $\left.\tau_{M}^{*} H^{*}(B U ; Q)\right)$ where $\tau_{M}: M \rightarrow B S O$ (or BU) classifies the tangent bundle of $M$;

(c) $R^{*}$ is its own annihilator $R^{* \perp}$, where

and

$$
R^{* \perp}=\left\{x \in H^{*}(M ; Q) \mid\langle x \cup y,[M]\rangle=0 \quad \forall y \in R^{*}\right\} ;
$$

(d) (in the oriented case only) if $M^{\prime} \subset M$ is an open-closed submanifold (union of components) with the unit class $1_{M^{\prime}} \in R^{\circ}$, then $M^{\prime}$ is an unoriented boundary.

The analogous result for mod 2 cohomology of closed manifolds was proved in [1].

The authors are indebted to the National Science Foundation for financial support during this work.

2. Proof of the Proposition. It is easy to see that $i^{*} H^{*}(V ; Q)$ satisfies (a)-(d). Specifically, (b) follows from $\tau_{M}=\tau_{V} \circ i$, (c) is a standard consequence of Poincaré-Lefschetz duality, and (d) is obtained by noting that each $M^{\prime}$ is the boundary of some union of components of $V$. The other implication is the difficult one.

Lemma. Let $X$ be a finite complex and $R^{*} \subset H^{*}(X ; Q)$ a homogeneous subalgebra with unit. Then $X$ decomposes uniquely into the disjoint union of

Received by the editors November 2, 1972.

AMS (MOS) subject classifications (1970). Primary 57D90.

Key words and phrases. Cobordism.

(C) American Mathematical Society 1973 
open-closed subsets $X_{1} \cup \cdots \cup X_{r}$ so that $R^{\circ}$ has a basis given by the unit classes $1_{X_{1}}, \cdots, 1_{X_{r}}$.

Proof. Suppose $X$ is the disjoint union $Y_{1} \cup \cdots \cup Y_{s}$ where each $Y_{i}$ is an open-closed subset of $X$ and $e=\alpha_{1} 1_{Y_{1}}+\cdots+\alpha_{s} 1_{Y_{s}} \in R^{\circ}$ where the $\alpha_{i}$ are distinct rationals. Then $e^{p}=\alpha_{1}^{p} 1_{Y_{1}}+\cdots+\alpha_{s}^{p} 1_{Y_{s}}$ is in $R^{\circ}$ for $p=$ $0,1, \cdots, s-1$. Since the Vandermonde determinant is nonsingular, each $1_{Y_{i}}$ lies in the span of $e^{\circ}=1, e, \cdots, e^{s-1}$, and hence in $R^{\circ}$.

Lemma. Let $X$ be a finite complex and $R^{*} \subset H^{*}(X ; Q)$ a homogeneous subalgebra with unit. There is a torsion free space $Y$ and a continuous map $f: X \rightarrow Y$ so that $f^{*} H^{*}(Y ; Q)=R^{*}$ and so that $f^{*}: H^{i}\left(Y ; Z_{2}\right) \rightarrow H^{i}\left(X ; Z_{2}\right)$ is the zero homomorphism for all $i>0$.

Proof. By the previous lemma, one may decompose $X$ into $X_{1} \cup \cdots \cup$ $X_{r}$, with $\left\{1_{X_{i}}\right\}$ a base for $R^{\circ}$. Then $1_{X_{i}} \cdot R^{*}=R^{*} \cap H^{*}\left(X_{i} ; Q\right)$ decomposes $R^{*}$ as a direct sum. If one finds appropriate maps for each $X_{i}$ to realize $1_{X_{i}} \cdot R^{*}$, the disjoint union will give the desired map for $X$. Thus, one may suppose $R^{\circ} \simeq Q$ with base the unit class 1 . Let $n$ be the dimension of $X$. For any class $x \in H^{j}(X ; Q), j>0$, there is an integer $m_{x} \neq 0$ and class $x^{\prime} \in H^{j}(X ; Z)$ so that $\rho_{Q} x^{\prime}=m_{x} x$, where $\rho_{Q}$ denotes rational reduction. Letting $s>n$, one may find a map $g_{x}: X \rightarrow K(Z, j)^{s}$ into the $s$-skeleton of an Eilenberg-Mac Lane space so that $g_{x}^{*}\left(i_{j}\right)=2 x^{\prime}$, where $i_{j}$ denotes the fundamental class.

There is a class $\sigma_{j} \in K^{*}\left(K(Z, j)^{s}\right)$ for which $\operatorname{ch}\left(\sigma_{j}\right)=p_{x} \rho_{Q}\left(i_{j}\right)+$ higher terms, where $p_{x} \neq 0$ is an integer, ch being the Chern character. In particular, there is a map $\phi_{j}: K(Z, j)^{s} \rightarrow Y_{j}$, where $Y_{j}=B U$ for $j$ even or $U$ for $j$ odd so that $\phi_{j}^{*}\left(\mathrm{ch}_{j}\right)=p_{x} \rho_{Q}\left(i_{j}\right)$, where $\mathrm{ch}_{j} \in H^{j}\left(Y_{j} ; Q\right)$ is the $j$-dimensional component of the Chern character.

Now let $x_{i} \in H^{j_{i}}(X ; Q), j_{i}>0$, be a set of generators for $R^{*}$. Let $Y$ be the product $\prod Y_{j_{i}}$ of the corresponding $Y_{j}^{\text {'s }}$ and $f: X \rightarrow Y$ the product $\prod \phi_{j_{i}} \circ g_{x_{i}}$ of the maps corresponding to $x_{i} . Y$ is connected, since each $Y_{j_{i}}$ is connected so $f^{*} H^{\circ}(Y ; Q)=R^{\circ}$ and similarly $Y$ is torsion free. Since $g_{x_{i}}^{*^{2}}\left(i_{j_{i}}\right)=2 x^{\prime}, g_{x_{i}}^{*}$ is zero on positive dimensional mod 2 cohomology, and hence $f^{*}$ is also. Then $\left(\phi_{j_{i}} \circ g_{x_{i}}\right) *\left(\mathrm{ch}_{j_{i}}\right)=2 p_{x_{i}} m_{x_{i}} \cdot x_{i}$, so $f^{*} H^{*}(Y ; Q) \supset R^{*}$ and $\operatorname{im} f^{*} \subset \operatorname{im}\left(\prod g_{x_{i}}\right) * \subset R^{*}$ so $f^{*} H^{*}(Y ; Z)=R$.

Now let $M^{n}$ be a closed oriented (or s.a.cx.) manifold, with $R^{*} \subset$ $H^{*}(M ; Q)$ satisfying conditions (a)-(d). Let $f: M \rightarrow Y$ be the map given by the Lemma so that $f^{*} H^{*}(Y ; Q)=R^{*}$.

For $u \in H^{j}(Y ; Q)$ and $v \in H^{n-j}(B S O ; Q)$ (or $\left.H^{n-j}(B U ; Q)\right), f^{*}(u) \in R^{*}$ and $\tau_{M}^{*}(v) \in R^{*}=R^{*} \perp$, and so $\left\langle\tau_{M}^{*}(v) \cup f^{*}(u),[M]\right\rangle=0$, so that all Pontrjagin (or Chern) numbers of $f: M \rightarrow Y$ are zero. Further, in the oriented case, for all $u \in H^{j}\left(Y ; Z_{2}\right)$ and $v \in H^{n-j}\left(B S O ; Z_{2}\right),\left\langle\tau_{M}^{*}(v) \cup f^{*}(u),[M]\right\rangle=0$ 
(if $j>0, f^{*}(u)=0$, while for $j=0, f^{*}(u)$ is a sum of classes $1_{M_{i}}$, where $M=M_{1} \cup \cdots \cup M_{r}$ is the decomposition given by $R^{\circ}$ and this is a StiefelWhitney number of the corresponding union of the $M_{i}$-which is zero since each $M_{i}$ is an unoriented boundary), and so all Stiefel-Whitney numbers of $f: M \rightarrow Y$ are zero.

Since $Y$ is torsion free, characteristic numbers determine the bordism class, and $[M, f]$ is zero in $\Omega_{n}^{S O}(Y)$ (or $\Omega_{n}^{U}(Y)$ ). Thus, there is a compact oriented (or s.a.cx.) manifold with boundary $V$ having $\partial V=M$ and map $F: V \rightarrow Y$ so that $f=F \circ i$.

Then $R^{*}=f^{*} H^{*}(Y ; Q)=i^{*} F^{*} H^{*}(Y ; Q) \subset i^{*} H^{*}(V ; Q)$ and taking annihilators, $R^{*}=R^{* \perp} \supset i^{*} H^{*}(V ; Q)^{\perp}=i^{*} H^{*}(V ; Q)$. Thus $V$ is the required manifold with boundary.

\section{REFERENCE}

1. R. E. Stong, Bilinear forms on manifolds, Math. Ann. (to appear).

Department of Mathematics, Yale University, New Haven, Connecticut 06520

Department of Mathematics, University of Virginia, Charlottesville, Virginia 22903 (Current address of R. E. Stong)

Current address (Larry Smith): Matematisk Institut, 8000 Aarhus C, Denmark 